\title{
Féeries
}

Études sur le conte merveilleuX, XVII ${ }^{\text {-XIXe }}$ siècle

$10 \mid 2013$

Conte et croyance

\section{Le statut paradoxal du conte philosophique : construction et déconstruction des croyances dans trois contes de Diderot}

The Paradoxical Status of the Philosophical Tale: Construction and

Deconstruction of Beliefs in Three Tales by Diderot

\section{Magali Fourgnaud}

\section{OpenEdition \\ Journals}

Édition électronique

URL : http://journals.openedition.org/feeries/902

DOI : $10.4000 /$ feeries.902

ISSN : 1957-7753

Éditeur

UGA Éditions/Université Grenoble Alpes

\section{Édition imprimée}

Date de publication : 20 septembre 2013

Pagination : 233-252

ISBN : 978-2-84310-253-0

ISSN : $1766-2842$

\section{Référence électronique}

Magali Fourgnaud, «Le statut paradoxal du conte philosophique : construction et déconstruction des croyances dans trois contes de Diderot », Féeries [En ligne], 10 | 2013, mis en ligne le 20 mars 2015, consulté le 08 septembre 2020. URL : http://journals.openedition.org/feeries/902 ; DOI : https:// doi.org/10.4000/feeries.902 


\section{LE STATUT PARADOXAL DU CONTE PHILOSOPHIQUE : CONSTRUCTION ET DÉCONSTRUCTION DES CROYANCES DANS TROIS CONTES DE DIDEROT}

$R$ OMANS ", "CONTES DE VIEILLES ", "fictions", "fables", telles sont les expressions que les philosophes des Lumières emploient pour désigner toute forme de croyance, au sens d'opinion énoncée sans examen, sans vérification. De nombreux articles du Dictionnaire philosophique (I764) de Voltaire soulignent cette opposition entre les lumières de la raison, «l'esprit philosophique», et l'égarement de la fiction narrative, notamment de la

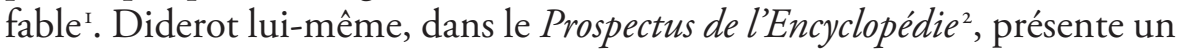
système de connaissances humaines fondé sur la distinction entre la raison, et donc la philosophie, et l'imagination, et donc la poésie définie comme fiction ${ }^{3}$. La force du raisonnement que produit l'esprit philosophique ${ }^{4}$, et qui permet de distinguer le bon du mauvais, le vrai du faux, semble a priori bien éloignée du conte, que D’Alembert définit comme « une histoire fausse $\&$ courte qui n'a rien d'impossible ${ }^{5}$. Considéré de la sorte, le conte, pris au sens large, se place du côté de l'illusion, et non du côté de la raison et de la clairvoyance. Pour autant, la fiction n'est-elle pas susceptible de transmettre un savoir de vérité ? En effet, un discours théorique, qui chercherait à remettre en question une opinion admise pour vraie, court le risque d'être considéré lui-même comme l'expression d'une croyance. En revanche, la fiction narrative, qui nécessite l'adhésion du lecteur, peut, selon nous, lui

I. Voir entre autres, l'article «Préjugé» de Voltaire, dans R. Naves (éd.), Dictionnaire philosophique, Paris, Garnier, 2008, p. 331.

2. Diderot, "Système des connaissances humaines», Prospectus de l'Encyclopédie, dans J. Lough et J. Proust (éd.), Euvres complètes, t. V, Paris, Hermann, I976, p. I05.

3. Ibid., p. II7.

4. Le Chevalier de Jaucourt, article "Philosophique (esprit)», Encyclopédie ou dictionnaire raisonné des sciences, des arts et des techniques, vol. II, t. I2, Paris, Briasson/David/Le Breton/Durand, I752, p. 515 .

5. D’Alembert, article «Conte, fable, roman», ibid., vol. I, t. IV, p. III. 
faire prendre conscience des mécanismes du processus de créance. Dans ce cas-là, elle acquerrait un statut paradoxal et deviendrait à la fois l'objet et le moyen de la réflexion. Cherchant à définir la croyance religieuse, l'abbé Mallet, dans l'article qu'il lui consacre dans l'Encyclopédie, reconnaît la force persuasive du récit. Il distingue «l'évidence d'objet ${ }^{6}$ ", établie par raisonnement et observation, qui caractérise la science et la connaissance, et l' "évidence de témoignage», comprise comme un consentement à une proposition, fondé seulement sur l'autorité ou le témoignage de quelques personnes qui assurent la vérité d'un fait. Notre esprit peut adhérer à une proposition, la considérer comme vraie, même si elle n'est passée ni par l'épreuve de nos sens, ni par celle de notre entendement; et cette idée, qui peut s'appuyer sur un récit, devient une certitude. Ce qui conduit l'abbé Mallet à conclure qu' "il y a une très grande différence entre cette proposition, ce que l'on doit croire est évident, \& celle-ci, il est évident qu'on doit croire telle chose ${ }^{7}$ ». Il distingue donc le contenu de la croyance (qui peut être remis en cause) et l'évidence qui nous pousse à adhérer à une proposition, sentiment d'évidence, qui dépasse à la fois l'expérience des sens et toute rationalité. Dans ces conditions, la réflexion philosophique sur la croyance (ses sources, ses mécanismes, ses conséquences,...) peut-elle se satisfaire d'une forme rationnelle et théorique? Le conte philosophique, qui cherche à susciter l'adhésion du lecteur, tout en démontant les croyances, apparaît comme un lieu tout à fait approprié pour s'interroger sur ce sujet : il se présente comme une expérience de pensée, à même de nous faire expérimenter le processus de créance tout en nous en montrant les mécanismes.

Dans le numéro de la revue Féeries consacré au conte oriental, JeanFrançois Perrin a montré que le succès de ce genre au début du XvıII ${ }^{\mathrm{e}}$ siècle exprimait un profond désir d'émancipation des esprits, le détour par l'Autre étant un moyen de questionner, voire de remettre en cause les carcans de la société :

La machinerie narrative des recueils imités des Nuits produit [...] des récits tendanciellement laïcisés en instruments d'investigation et de critique de la croyance par les croyances, de la coutume par les coutumes - last but not least, de l'empire de la fiction par les fictions elles-mêmes ${ }^{8}$.

Cette interrogation sur ce qui nous pousse à adhérer à une proposition, jusqu'à l'aveuglement parfois, apparaît non seulement comme une

6. L'abbé Mallet, article «Croyance», ibid., vol. I, t. II, p. 516.

7. Ibid., p. 517.

8. J.-F. Perrin, «L'invention d'un genre littéraire au XviII ${ }^{\mathrm{e}}$ siècle», Féeries, nº 2, 2005, p. 27. 
caractéristique du conte oriental, mais aussi comme un indice générique du conte à visée morale et philosophique. En effet, au tournant des XVII ${ }^{\mathrm{e}}$ et $\mathrm{XVIII}^{\mathrm{e}}$ siècles, émerge une sous-catégorie du conte dont l'intention affichée est d'éclairer les lecteurs sur les causes du malheur des hommes, à savoir l'imagination et les illusions, comme l'explique Marmontel lui-même dans la préface de ses contes moraux :

Je me proposai d'y faire sentir la folie de ceux qui emploient l'autorité pour mettre une femme à la raison; et je pris pour exemple un sultan et son esclave, comme les deux extrémités de la domination et de la dépendance. [...] L'idée singulière que les jeunes personnes se font de l'amour, d'après la lecture des romans, et le chagrin qu'elles ont de ne pas le trouver dans la nature tel qu'il est peint dans les livres, était un petit ridicule à combattre?.

Il s'agit, pour Marmontel, de rendre le lecteur plus lucide en mettant en lumière ce qui aveugle les hommes, et partant de le rendre plus tolérant sur les faiblesses humaines. Mais comment le conte à visée morale et philosophique peut-il démonter l'illusion tout en maintenant le charme qui le lie à son lecteur? Quels dispositifs narratifs peuvent susciter à la fois l'adhésion et la réflexion distanciée? L'ensemble des contes à visée morale et philosophique présente, plus ou moins explicitement, une structure enchâssée qui leur confère une forte dimension métatextuelle. Certes la composition emboîtée n'est pas récente, elle remonte au moins au Décameron de Boccace, se poursuit par l'Heptaméron de Marguerite de Navarre; Les Mille et Une Nuits elles-mêmes, traduites par Galland en I704, utilisent le même dispositif narratif. Mais ce dernier prend, au début $\mathrm{du} \mathrm{XVIII}^{\mathrm{e}}$ siècle, une fonction morale et philosophique. L'autoréflexivité fait entrer le lecteur dans le laboratoire de la fiction, le conduit à assister à la création de la mystification : le récit-cadre, qui présente le conteur et ses auditeurs, fictionnalise la situation du lecteur lui-même. Cet effet d'analogie conduit ce dernier à s'interroger à la fois sur ce qu'il voit et sur ce qu'il croit. Les contes de Diderot montrent particulièrement bien ce rapport paradoxal de proximité et de prise de distance de la fiction, comme en témoignent les trois textes ${ }^{10}$ qu'il s'agit ici d'étudier : Les Bijoux indiscrets (1748) qui présentent un cadre oriental, puis l'Entretien d'un père avec ses enfants ou du danger de se mettre au-dessus des lois et Les Deux Amis de Bourbonne, tous deux édités pour la première fois dans un recueil de

9. J.-F. Marmontel, Contes moraux, par M. Marmontel; suivis d'une Apologie du théâtre, La Haye, I76I, préface, p. V.

IO. Notre édition de référence est la suivante : Contes et romans, M. Delon (éd.), Paris, Gallimard, 2004. 
Contes moraux et nouvelles idylles de Salomon Gessner en allemand en 1772 et en français en I773. Alors que le premier semble suivre la mode du conte libertin, parodique et anticlérical, les deux autres présentent une esthétique plus "réaliste», a priori éloignée de l'univers fantaisiste du conte oriental et semblent exprimer la philosophie du droit naturel, fondée sur la raison et l'empathie. Il y aurait donc une évolution des œuvres narratives de Diderot, qui "part du conte philosophique pour arriver à la philosophie par le conte ${ }^{\mathrm{II}} »$. Nous souhaiterions montrer que cette philosophie s'exprime bel et bien dès le début dans la structure narrative, que reprennent les récits ultérieurs. En effet, ces trois contes ont en commun une structure enchâssée et grâce à cette composition, le lecteur se laisse emporter par l'histoire, mais conserve néanmoins un recul critique. Ainsi, par ce double mouvement, le lecteur du conte vit une expérience similaire à celle du spectateur de théâtre : il perçoit les ficelles d'une double mystification, celle du personnage et la sienne (on sait combien Diderot utilise le procédé comme un moteur narratif ${ }^{\mathrm{12}}$ et comme un déclencheur de la réflexion ${ }^{13}$, dans le texte intitulé lui-même Mystification et dans $L a$ Religieuse). En favorisant à la fois l'adhésion du lecteur dans l'histoire et sa réflexion critique, le conte se présente comme une "feintise partagée ${ }^{14}$ ». Comme l'a montré Jean-Marie Schaeffer, ce dispositif est un opérateur cognitif, il est susceptible de transmettre un savoir, notamment sur les processus de la croyance.

La composition des Bijoux indiscrets fait apparaitre quatre niveaux narratifs. Une voix se fait entendre, qui n'est ni celle de l'auteur, ni celle du narrateur, mais celle d'une instance qui annonce voire commente le récit qui va suivre : c'est elle qui découpe le récit en deux parties (tome premier/ tome second) et donne un titre aux chapitres. Ces interventions coupent la fluidité de la lecture et ont une fonction métatextuelle, comme l'indiquent les jeux sur les titres. Ces derniers décomptent rigoureusement les essais de l'anneau, mais oublient de mentionner le vingtième (on passe directement du dix-neuvième essai du chapitre XXXIX au vingtet-unième au chapitre XLI). Cette omission, «loin d'être involontaire ou de résulter d'une transcription, est tout simplement l'aveu délibéré (ou la signature) d'une mystification très caractéristique de l'homme et du

II. J.-P. Seguin, Diderot, le discours et les choses : essai de description du style d'un philosophe en I750, Paris, Klincksieck, I978, p. I59.

I2. R. Kempf, Diderot et le roman ou le démon de la présence, Paris, Seuil, I984, p. 220-221.

I3. P. Chartier, "Diderot, ou le rire du mystificateur ", Dix-huitième siècle, n 32, 2000, p. I45-164.

I4. J.-M. Schaeffer, Pourquoi la fiction?, Paris, Seuil, 1999, p. 156. 
philosophe ${ }^{15}$ ". Au deuxième niveau narratif, un narrateur s'adresse à Zima, au début du premier tome, et l'invite à lire un ouvrage licencieux dont le titre est Les Bijoux indiscrets. Le narrateur de ce roman se présente comme le traducteur d'un auteur africain, qui souligne lui-même son rôle dans l'élaboration de l'histoire : il découpe, déplace, trie les événements de l'histoire de Mangogul. Le troisième niveau est consacré à l'histoire du sultan et de sa favorite, le premier possédant un anneau qui a le pouvoir de le rendre invisible et de faire parler les sexes féminins. Se trouvent alors insérées les histoires libertines des mondaines et des couventines qui correspondent au quatrième niveau narratif. Sélim, le courtisan du sultan, raconte à son tour sa propre histoire (chapitres XLIV et XLVI) et y insère l'apologue d'Hilas et d'Iphis (réécriture de Tanzaï et Néardané, de Crébillon). Cette structure enchâssée a donc pour effet de déconstruire toute illusion romanesque et de mettre en lumière les ficelles de sa construction : le conte démolit sa propre capacité à créer l'illusion et devient dès lors un laboratoire où se montent et se démontent toutes les formes de croyances et de persuasion voire de séduction (de la religion, de la science, des discours amoureux, de la fiction elle-même).

En effet, les bijoux féminins sont censés révéler ce qui devait rester caché, dire la vérité sur les comportements des femmes, en particulier sur leurs infidélités. Comme dans Le Sopha, conte moral (1742) de Crébillon, le dispositif permet de dévoiler les coulisses de la comédie sociale, de mettre au jour ce qui se cache derrière les masques mondains. En ce sens, Mangogul offre bien aux siècles ultérieurs des sujets d' "incrédulité ${ }^{16}$ ", entendue comme dénonciation des hypocrisies sociales. Le leurre des bijoux parlants a effectivement des conséquences dans toutes les sphères de la société et il permet notamment de tourner en dérision l'adhésion irraisonnée aux superstitions et aux oracles. L'ironie du narrateur souligne l'incompétence du prêtre Codindo, qui «ne savait non plus lire aux astres que vous et [lui] ${ }^{17}$ ». Le portrait du génie Cucufa, l'instigateur de la mystification, amalgame les attributs de la sorcellerie et des religions catholique et hindoue : il porte la tenue vestimentaire des cordeliers; tel un sorcier, il est accompagné d'un hibou, de rats et de chauve-souris; il suit l'office des bramines; il est présenté comme un «camaldule», francisation de l'italien

I5. J. Rustin, «Préface» aux Bijoux indiscrets, p. 25. Cité par M. Delon, note 3, dans Contes et romans, ouvr. cité, 2004 , p. 956.

16. Les Bijoux indiscrets, ouvr. cité, p. I31.

17. Ibid., p. 6. 
camaldoli $^{18}$. Le portrait syncrétique se clôt par l'attaque ironique du narrateur qui le désigne comme un "cafard ${ }^{19}$ ". La description des muselières qu'imposent les religieux, afin de se protéger des révélations scandaleuses des bijoux, est l'occasion d'une satire féroce visant la religion liberticide : le chapitre VIII décrit le sort des couventines et annonce, sur le mode burlesque, les dénonciations de L'Oiseau blanc, conte bleu ${ }^{20}$ et de La Religieuse. L'ironie du narrateur souligne l'absurdité des religieux à vouloir trouver une origine divine au babillage des bijoux :

La religion revendiqua leur caquet comme une matière de sa compétence, et ses ministres prétendirent que le doigt de Brama se manifestait dans cette œuvre. Il y eut une assemblée générale des pontifes, et il fut décidé qu’on chargerait les meilleures plumes de prouver en forme que l'événement était surnaturel, et qu'en attendant l'impression de leurs ouvrages, on le soutiendrait dans les thèses, dans les conversations particulières, dans la direction des âmes, et dans les harangues publiques ${ }^{21}$.

Le passage montre comment naît et se diffuse la croyance, qui se distingue ici de la foi, et comment elle se fonde uniquement sur du discours : il s'agit d'une évidence de témoignage et non d'objet, selon la distinction de l'abbé Mallet. La parole d'autorité des religieux, adressée à des masses et proférée dans des lieux sacrés, suffit pour faire croire à l'origine divine du phénomène. Au chapitre XV, le récit de la harangue du bramine et de ses effets sur son auditoire met en évidence les procédés rhétoriques de la persuasion (appel aux sentiments, déclenchement de la terreur et de la pitié) et de leur inefficacité sur le plan moral : «Le reste de l'auditoire le regarda comme un prophète, versa des larmes, se mit en prières, se flagella même, et ne changea point de vie ${ }^{22}$.» Telle est également la réaction de Mme de*** en écoutant le récit d'Olivier et de Félix dans Les Deux Amis de Bourbonne: l'histoire l'émeut profondément, mais la lettre de M. Papin, docteur en théologie, a raison de ses sentiments et parvient à la convaincre de l'impiété des deux amis. Les larmes ne modifient en rien son comportement. Â l'écoute du sermon du bramine, seuls le sultan et la favorite restent incrédules et indifférents : «Mangogul et la sultane, qui

18. "Certain ordre de religieux établis en un désert, fondé par saint Romuald, qui ont pris leur nom du lieu où ils sont établis» (Furetière), cité par M. Delon, note 3, dans Contes et romans, ouvr. cité, p. 925 .

19. Les Bijoux indiscrets, ouvr. cité, p. I2.

20. «Les premières cibles de Diderot, dans le conte, sont la divinité, les dogmes, les religieuses, les prêtres, transformés ici en génies infernaux et sadiques. ", A. Defrance, notice de L'Oiseau blanc, conte bleu, dans Contes, A. Defrance et J.-F. Perrin (éd.), Paris, Honoré Champion, 2008, p. I435.

21. Les Bijoux indiscrets, ouvr. cité, p. 40.

22. Ibid., p. 43. 
seuls avaient le secret de l'anneau, trouvèrent que le bramine avait aussi heureusement expliqué le caquet des bijoux par le secours de la religion, qu'Orcotome par les lumières de la raison ${ }^{23}$.» La religion et la science sont ainsi renvoyées dos à dos, manière de dénoncer les charlataneries des pseudo-savants, comme Orcotome qui se nomme médecin des bijoux, grand expert de ce qui lui échappe complètement. Le pouvoir magique de l'anneau se donne alors pour fonction de «rabattre la présomption de l'empirique ${ }^{24}$ ", selon l'expression du sultan lui-même qui prend un malin plaisir à déjouer les affirmations dogmatiques d'Orcotome. L'ironie du narrateur souligne également le décalage entre les prétentions du savant et son ignorance : "Orcotome venait de répondre à beaucoup de choses, mais il croyait avoir satisfait à tout : il se trompait ${ }^{25}$." Le chef de file des "vorticoses", c'est-à-dire des partisans des tourbillons, disciples de Descartes, se nomme significativement "Persiflo", réduisant le raisonnement scientifique à un persiflage ${ }^{26}$ : il raisonne "comme un bijou ${ }^{27}$ ». La mystification initiale renverse donc tous les discours d'autorité (religieux, scientifiques, juridiques), qui prétendent détenir la vérité.

Le lecteur prend en effet conscience des stratégies de manipulation, notamment des filouteries des vendeurs de muselières, sorte de ceintures de chasteté capables de faire taire les bijoux. Le personnage d'Éolipile (chapitre XVII), le bijoutier le plus célèbre, n'est pas sans rappeler le cynique Filerin, profiteur des faiblesses humaines, dans L'Amour médecin, de Molière. Même les témoignages dans le cadre d'un procès sont soupçonnés de mensonge. L'anneau de Mangogul permet par exemple de libérer Kersael, accusé à tort de viol par sa maîtresse (chapitre XXVIII) : «Il est inouï [...] que la simple allégation d'une accusatrice suffise pour mettre en péril la vie d'un citoyen $^{28}$.» Dès lors, l'anneau trouve une utilité morale : Mirzoza reproche au sultan de l'avoir utilisé uniquement pour ses plaisirs, en revanche, elle l'encourage à l'utiliser pour la découverte de la vérité et le bonheur de ses sujets. C'est l'ensemble des déclarations qui se trouve emporté dans une ère du soupçon, y compris celles d'amour et de fidélité. Croire à l'amour sincère, c'est, pour Mangogul, croire aux contes de fées et avoir l'esprit gâté par les romans : "Vous avez vu là des héros respectueux et des princesses

23. Ibid.

24. Ibid., p. 7I.

25. Ibid., p. 28.

26. E. Bourguignat, Le Siècle du persiflage, I734-I789, Paris, PUF, 1998, p. 64.

27. Les Bijoux indiscrets, ouvr. cité, p. 25.

28. Ibid., p. 93. 
vertueuses jusqu'à la sottise, et vous n'avez pas pensé que ces êtres n'ont jamais existé que dans la tête des auteurs ${ }^{29}$ ", dit-il à la favorite.

Par conséquent, en montrant les ficelles de la comédie humaine, la fiction transmet une vérité, comprise comme envers de l'illusion. En effet, elle met en lumière le mécanisme des comportements des hommes, assimilés à des marionnettes. Tel est le sens de l'apologue que raconte Sélim au chapitre L. Il présente les courtisans comme les pantins d'une fée qui les manipule (on y a vu une allégorie de Madame de Maintenon), ce qui n'est pas sans évoquer le conte de Voltaire, Pot-pourri (1765), où Jésus est assimilé à Polichinelle ${ }^{30}$. Son récit s'apparente à un conte étiologique : la forme des robes trouve son origine au temps où on marchait sur la tête. En ce sens, le conte inséré de Sélim se présente comme une métonymie de l'ensemble du conte-roman. En effet, en donnant la parole au bas corporel, le conte de Diderot s'inscrit dans la tradition du comique carnavalesque, tel que l'a théorisé Mickaël Bakhtine ${ }^{31}$ : il s'agit de renverser les hiérarchies préétablies, de remettre en cause l'autorité des discours dominants et la certitude en une vérité unique et immuable. Le conte lui-même se prend pour cible de sa propre critique. Selon Mangogul, les bijoux ont le pouvoir de révéler la vérité, de distinguer le vrai du faux, comme l'affirme le bijou d'Amine :

Oh! que ces hommes sont sots de croire que des dignités, des honneurs, des titres, des noms, des mots vides de sens, en imposent à des bijoux! Chacun a sa philosophie, et la nôtre consiste principalement à distinguer le mérite de la personne, le vrai mérite, de celui qui n'est qu'imaginaire ${ }^{32}$.

Mais comment accorder foi à un tel propos? Dans le cadre d'un récit libertin, le lexique religieux (autel, sacrifice) et moral (mérite, vertu) est détourné et prend une connotation sexuelle. En outre, les bijoux sont considérés euxmêmes comme des oracles par le sultan, qui croit leurs récits sans aucune distance critique, ce que lui reproche la favorite :

Tant qu'ils auront la prudence de ne parler que de ce qu'ils entendent, je les croirai comme des oracles. - On pourrait, dit Mirzoza, en consulter de plus sûrs. - Madame, reprit Mangogul, quel intérêt auraient ceux-ci de déguiser la vérité? Il n'y aurait qu'une chimère d'honneur qui pût les y porter; mais un bijou n'a point de ces chimères. Ce n'est pas là le lieu des préjugés. — Une chimère d'honneur! dit Mirzoza, des

29. Ibid., p. 213.

30. Voltaire, Pot-pourri, dans S. Menant (éd.), Contes en vers et en prose, Paris, Bordas, I993, p. 42.

31. M. Bakhtine, L'Euvre de François Rabelais et la culture populaire au Moyen Âge et sous la Renaissance, Paris, Gallimard, 1970.

32. Les Bijoux indiscrets, ouvr. cité, p. 176. 
préjugés! Si votre hautesse était exposée aux mêmes inconvénients que nous, elle sentirait que ce qui intéresse la vertu, n'est rien moins que chimérique ${ }^{33}$.

Le vieillard tahitien ne dira pas autre chose à Bougainville : "Laisse-nous nous reposer; ne nous entête ni de tes besoins factices, ni de tes vertus

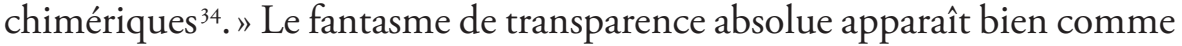
une chimère, comme la marotte du sultan, sur laquelle il fonde son pouvoir. Mirzoza souligne : "Voilà donc, reprit la sultane, où se réduisent vos longues études et toute votre philosophie, à supposer un fait, et à l'appuyer sur des expressions populaires ${ }^{35}$.» C'est présenter à son tour le discours des bijoux, censés délivrer la vérité, comme l'expression d'une croyance. De fait, leurs propos sont incomplets (le bijou de Fanni reconnaît avoir une mémoire défectueuse) et ne font que répéter ce que tout le monde sait déjà. Mangogul reconnait que «les bijoux sont de petits fous qui ne savent ce qu'ils disent. La bague de Cucufa peut les faire parler, mais non leur arracher la véritée ${ }^{36}$ ». Sélim souligne également le caractère partiel du persiflage des bijoux et les complète en faisant le récit de ses propres conquêtes. Le babillage des bijoux déclenche un flot de paroles et d'interprétations : "c'était un texte inépuisable. Aux faits véritables, on en ajoutait de faux; tout passait : le prodige avait rendu tout croyable ${ }^{37}$. " Seule la favorite reste incrédule aux propos qui lui sont tenus, elle est la seule à distinguer le mot de la chose : "On me dirait une chose assez obscure, à laquelle je ne répondrais que par un fait d'expérience ${ }^{38}$.» Elle distingue en ce sens l'objet et le discours porté sur l'objet, "l'évidence d'objet» et «l'évidence de témoignage», selon les expressions de l'abbé Mallet.

Paradoxalement, seule la fiction semble capable de dessiller les yeux des hommes. En effet, comme dans «Le Pouvoir des fables ${ }^{39}$ » de La Fontaine, c'est le conte grivois de Mangogul qui parvient à résoudre le conflit absurde qui oppose les deux pontifes (se querellant autour de la question du penum, qui n'est pas sans rappeler la parodie de l'affaire de la Bulle Unigenitus dans Tanzaï et Néardané). Le sultan conduit les deux religieux à croire à son histoire extravagante en soulignant l'analogie entre les personnages et les personnes que connaissent ses auditeurs :

33. Ibid., p. 2I.

34. Le Supplément au voyage de Bougainville, dans Contes et romans, ouvr. cité, p. 549.

35. Les Bijoux indiscrets, ouvr. cité, p. 98.

36. Ibid., p. 205.

37. Ibid., p. 28.

38. Ibid., p. 100.

39. La Fontaine, Fables, livre huitième, fable IV, J.-C. Darmon (éd.), Paris, Le Livre de poche, 2002, p. 237-239. 
"Ce derrière blanc comme la neige, gras, ramassé, arrondi, joufflu, potelé, ressemblait, comme deux gouttes d'eau, à celui de la femme du souverain pontife.»

Le pontife : De ma femme!

Le sultan : Pourquoi pas? Le personnage aux deux trous ajouta : "C'était elle en effet; car je me la remets ${ }^{40}$."

Cette illusion référentielle permet aux auditeurs de croire à l'histoire : leur adhésion est une condition nécessaire pour qu'opère la «leçon». Le sultan se sert en effet de la chute (ce n'était qu'un rêve) pour faire prendre conscience aux deux sectes de l'absurdité de leurs conflits religieux : le sultan leur fait remarquer que leur dogmatisme s'apparente à l'illusion des deux personnages dont ils ont bien ri et qu'ils prennent pour des fous. Au-delà de la grivoiserie, Mangogul apparaît comme un nouveau Socrate, piégeant ses auditeurs à leur propre logique. Le "rêve de Mangogul» se présente ainsi comme une mise en abyme du conte : il s'agit de piéger le lecteur amateur de contes libertins ou de récits à clefs, tout en lui faisant prendre conscience des mécanismes de l'illusion et donc de toute forme de duperie. En effet, le conte oriental de Diderot, comme le conte grivois du sultan, multiplie les échos à l'actualité la plus récente : les lecteurs contemporains ont pu reconnaître Madame de Pompadour sous les traits de Mirzoza et Louis XV sous ceux de Mangogul. Ils ont également reconnu la dispute opposant les ramistes aux lullistes dans la dispute entre Utrémifasolasiututut (Rameau) et Utmiutsol (Lully) (chapitre XIII), ainsi que les débats entre newtoniens et partisans des tourbillons dans le conflit confrontant les "attractionnaires », représentés par Circino ${ }^{4 \mathrm{I}}$ (Newton), aux "vorticoses», représentés par Olibri (Descartes) (chapitre IX). Toutes ces allusions à l'époque font des Bijoux indiscrets une véritable "œuvre de circonstance, non parce qu'elle aurait été rédigée en quelques heures et par défi, mais en ce qu'elle propose comme un instantané de l'aube des Lumières ${ }^{42}$ ", selon Michel Delon. Par conséquent, le lecteur est placé dans une situation similaire à celle des deux pontifes : le conte flatte dans un premier temps son imagination et sa curiosité, pour lui montrer ensuite les mécanismes de ses propres aveuglements. Mais la déconstruction de toutes les croyances peut avoir des conséquences dangereuses, ce que montre également le conte de Diderot : elle peut non seulement conduire à un scepticisme radical, mais également détruire le lien social.

40. Les Bijoux indiscrets, ouvr. cité, p. 45 .

4I. Du latin circinus, le compas.

42. M. Delon, Notice des Bijoux indiscrets, ouvr. cité, p. 9I8. 
En effet, la particularité des Bijoux indiscrets est de montrer les conséquences de l'illusion (sous-entendant la nécessaire lucidité capable de la déconstruire), tout en suggérant les effets ambigus de la quête absolue de transparence (comprise comme envers de l'illusion). L'anneau confère au sultan la capacité d'entendre ce qui devrait être tu et un pouvoir illimité à sa volonté ${ }^{43}$. Cette omnipotence et cette omniscience mettent mal à l'aise Mirzoza qui affirme qu'il s'agit d'un "secret diabolique ${ }^{44}$ » qui aura des «suites funestes ${ }^{45}$ ». L'anneau magique est de fait la source du désordre social, comme en témoignent la multiplication des charlatans et le développement de la calomnie, et c'est même un véritable instrument de torture, le sultan mettant les bijoux «à la question ${ }^{46}$ ». Le conte de Diderot montre, de l'intérieur, les mécanismes qui conduisent un prince libérateur à devenir un despote ${ }^{47}$ (au début du conte, il fait confiance aux femmes, brise les portes du harem, et en chasse les gardes). En effet, la folie et le soupçon généralisés viennent remettre en cause la confiance établie préalablement entre le roi et ses sujets : l'élément déclencheur est l'ennui du sultan et ses doutes quant à la fidélité de la favorite. Les Bijoux indiscrets apparaissent dès lors comme une réécriture du mythe de Gygès, tel qu'Hérodote le rapporte dans le livre I de son Histoire ${ }^{4}$. Le roi de Lydie, Candaule, persuade son esclave, Gygès, d'observer la reine nue, au moment du rituel du coucher, afin qu'il se rende compte de sa beauté. L'esclave a beau répondre à son maître qu'il s'agit d'une action perverse et contraire à la loi, Candaule parvient à le convaincre de l'absence de danger du stratagème. La reine se rend compte qu'elle a été vue, garde d'abord le silence, puis place Gygès face à un dilemme tragique : soit il doit tuer son maître, celui qui lui a donné le don de voir ce qui devait rester invisible, soit il doit se tuer lui-même pour avoir vu ce qu'il ne devait pas voir. Gygès tue son maître et fonde son pouvoir sur ce secret. Le mythe montre comment la perte de crédibilité de la parole du souverain conduit à une remise en cause du pouvoir luimême. En effet, Candaule décrédibilise sa parole, d'une part parce que dire

43. «Il n'avait qu'à mettre sa bague et dire : je veux être là; à l'instant il y était.» Ibid., p. I2.

44. Ibid., p. 43.

45. Ibid., p. I7-I8.

46. Ibid., p. I4. "Question signifie aussi, La torture, la gêne qu'on donne aux criminels, pour

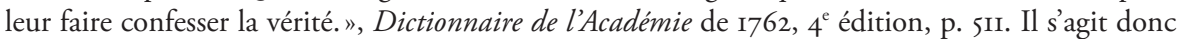
d'un instrument violent qui pousse à la confession et peut être lu comme une dénonciation de l'Inquisition.

47. La favorite «ne comptait pas assez sur les promesses d'un homme moins amoureux que despotique, pour être libre de toute inquiétude", Les Bijoux indiscrets, ouvr. cité, p. I7.

48. Hérodote, Histoire d'Hérodote, suivie de la vie d'Homère, nouvelle édition, t. I, A. F. Miot (éd.), Paris, Didot frères, livre I, chap. VII-XIV, p. 34-38. 
que la reine est belle ne suffit plus, il faut que l'esclave s'en rende compte de ses propres yeux. D'autre part, en invitant son esclave à outrepasser la loi, il met sa propre vie en danger, dans la mesure où, si transgresser est permis, le tuer et prendre sa place deviennent possibles. Dans son explication lumineuse du mythe, Louis Marin montre qu' «il y a une vérité dont le dévoilement doit rester voilé, pour qu'il y ait des regards qui voient, des visibles qui soient vus, des vérités qui soient communes et communicables, pour qu'il y ait une parole royale puissante qui fait être en disant ce qu'elle $\operatorname{dit}^{49}$ ». La transparence absolue a, elle aussi, des conséquences dangereuses, telle est donc une des «leçons» du conte de Diderot: Mangogul commet la même effraction du féminin et de l'intimité que Candaule. Certes, ce n'est pas sa vie qui est en péril, mais c'est la confiance que Mirzoza lui accorde. Elle le met d'ailleurs en garde régulièrement contre sa volonté d'assurance : "s'il [son bijou] vient à parler, je perdrai votre estime et votre cœur, et vous en serez au désespoir ${ }^{50}$ ». Ce qui pousse le sultan, et qui lui fait prendre des risques, ce n'est pas la volonté de remettre en cause les croyances et les préjugés, mais la quête irraisonnée de certitudes. Se demandant tout au long de l'histoire si Mangogul tiendra parole et si la favorite lui a été fidèle, le lecteur est lancé dans la même quête de certitude que le sultan. Il vit en ce sens le même "fantasme d'absolutisme ${ }^{5 \mathrm{I}}$ ", selon l'expression de Jean-Paul Sermain, pour ensuite prendre conscience de la vanité d'un tel désir. En effet, la construction déceptive ${ }^{52}$ du conte fait expérimenter au lecteur l'impossibilité d'un langage transparent et partant, d'un savoir absolu. Mangogul finit par faire l'essai de l'anneau sur la favorite, qui feint d'être endormie. Il ne voit pas qu'elle le voit, mais il lui affirme :

- [...] Je n'ai point fait sur vous l'essai de mon anneau; mais j'ai cru pouvoir, sans manquer à mes promesses, user d'une ressource qui vous rend à mes vœux et qui vous assure à mon cœur à jamais.

- Prince, dit la favorite, je vous crois; mais que l'anneau soit remis au génie, et que son fatal présent ne trouble plus ni votre cour, ni votre empire ${ }^{53}$.

Le conte se clôt donc sur une incertitude : la sultane croit-elle vraiment le sultan qu'elle vient de surprendre en train de rompre sa promesse? La

49. L. Marin, "Gygès», dans Lectures traversières, Paris, A. Michel, I992, p. I60.

50. Les Bijoux indiscrets, ouvr. cité, p. I3.

5I. J.-P. Sermain, «Le fantasme de l'absolutisme dans le conte de fées au XVIII ${ }^{e}$ siècle», Féeries, $\mathrm{n}^{\circ} 3,2006$, p. $75-85$.

52. T. M. Kavanagh, «Language as deception: Diderot's Les Bijoux indiscrets», Diderot Studies, vol. 23, I988, p. IOI-II3.

53. Les Bijoux indiscrets, ouvr. cité, p. 220 (je souligne). 
prospérité que leur annonce le génie se fonde sur un double secret, le silence de la favorite, qui ne dit pas au sultan qu'elle l'a vu la regarder, et le mensonge du sultan. À chacun de croire ce qu'il veut, les personnages comme le lecteur. Le conte souligne, de cette manière, à la fois la vanité et le danger d'une certitude absolue et l'impossibilité d'une concordance parfaite entre le langage et la chose, comme en témoignent les nombreuses lacunes du texte initial ${ }^{54}$. En ce sens, Les Bijoux indiscrets est bien, comme le souligne Colas Duflo, l'une « des rares fictions libertines, au sens fort, c'està-dire qui corresponde, comme Candide ou Justine, à ces trois critères : un récit expérimental, encyclopédique et surtout, philosophique"». En effet, le conte diderotien présente une triple particularité. Tout d'abord, la structure enchâssée permet d'emblée de déconstruire l'illusion romanesque, ce qui prémunit le conte contre toute accusation de feintise et l'éloigne des discours qui cherchent à susciter la croyance. Un tel discours de fiction, qui s'annule lui-même comme fiction, se présente dès lors comme le lieu par excellence du démontage de toutes les croyances et de tous les discours cherchant à persuader (discours religieux, scientifiques, amoureux). Enfin, la fiction devient elle-même objet de critique, soulignant également les dangers de la quête absolue de certitude. Le tour de force du conte philosophique est donc d'aboutir, par la fiction, à un discours qui n'est ni totalement fictionnel, ni théorique (ou alors qui est à la fois fictionnel et théorique), sur l'origine, les mécanismes et les conséquences de la croyance et de la quête de certitude.

Bien que présentant une esthétique plus « réaliste» que le conte oriental, l'Entretien d'un père avec ses enfants ou du danger de se mettre au-dessus des lois présente une composition similaire et soulève également une réflexion sur la croyance, entendue ici comme opinion. Dans ce conte, Diderot met en scène son propre père, entouré de ses trois enfants : Diderot luimême, à travers le personnage nommé «moi », sa sœur et son frère, l'abbé. La scénographie s'apparente de nouveau à une pièce de théâtre : en un moment unique, les personnages défilent dans un lieu unique, la chambre $\mathrm{du}$ vieillard. Ils sont tour à tour conteurs et auditeurs. De la même façon, Mirzoza anticipait la fin des récits des voyageurs de Mangogul et coopérait ainsi à la construction du récit ${ }^{56}$. Le père de famille commence par

54. Le narrateur souligne de manière récurrente les lacunes du texte initial : «ici l'ignorance des traducteurs nous a frustrés d'une démonstration que l'auteur africain avait conservée sans doute. À la suite d'une lacune de deux pages, ou environ, on lit [...].", ibid., p. 25.

55. C. Duflo, Diderot philosophe, Paris, Honoré Champion, 2003, p. 62.

56. «Mirzoza : Arrêtez, je vais continuer, donnèrent lieu à un schisme qui divisa les hommes, les femmes et tous les citoyens. Il y eut une insurrection d'école contre école, de maître contre maître; 
exposer son histoire (celle du testament du curé de Thivet) et son cas de conscience : devait-il brûler le testament et donner l'héritage aux descendants misérables du curé ou devait-il respecter la loi? Le personnage nommé «Moi» (le philosophe) interrompt le premier récit et rapporte ensuite l'anecdote du maire de la ville, de La Mésangère, qui déclenche une réflexion autour de la question suivante : doit-on soigner un criminel malade? Arrivent ensuite un ecclésiastique, un notaire, Dubois, et un chapelier. Ce dernier leur fait part à son tour de son histoire et de son cas de conscience : est-il juste qu'il bénéficie de l'héritage de sa femme, qu'il a soignée pendant dix-huit ans, bien que la loi le destine à ses descendants? Une fois le chapelier sorti, le prieur leur rapporte l'histoire du calzolaio de Messine, qu'il a lue dans l'ouvrage du père Labat (histoire du sicilien qui, déçu de voir les assassins impunis, décide de tenir une cour de justice dans son propre magasin et de tuer les coupables de ses propres mains). Enfin, lorsque $\mathrm{M}^{\mathrm{me}} \mathrm{d}$ 'Isigny, la voisine, arrive, le témoignage de sa vie déclenche un débat sur l'égalité des sexes et l'infidélité. Chaque histoire rapportée se présente ainsi comme un exemple des différents problèmes soulevés par la question du respect de la loi (thème qu'annonce le titre) : dans quelle mesure une action humaine, régie par l'empathie et la raison, peut-elle être généralisée et s'ériger en règle sociale? De ce fait, les récits s'apparentent à des modélisations, propres à susciter une réflexion d'ordre moral et philosophique. Le conte moral de Diderot, comme son conte oriental, emporte donc le lecteur dans un double mouvement : plongée dans la fiction et prise de distance.

En effet, les éléments renvoyant à la réalité contemporaine (les relations familiales, le personnage du notaire, Dubois, qui a réellement existé) et les "petits détails vrais» (le vieillard demande par exemple à la sœur du philosophe de remonter l'oreiller descendu trop bas) favorisent l'adhésion du lecteur : ces éléments qui rendent l'histoire vraisemblable et qui créent l'illusion romanesque le portent à croire à l'histoire. En revanche, la composition enchâssée du récit conduit le lecteur à prendre du recul. Comme dans le cas des Bijoux indiscrets, le récit-cadre rappelle les circonstances dans lesquelles chaque interlocuteur parle et montre les incohérences entre l'attitude et les propos : cette construction emboîtée crée un effet de distanciation, comme au théâtre. Le récit-cadre permet ainsi de distinguer un moi-narrant (celui qui se souvient) et un moi-narré (le jeune philosophe

on disputa, on s'injuria, on se haït. — Fort bien; mais ce n'est pas tout. — Aussi n'ai-je pas tout dit... - Achevez... ", Les Bijoux indiscrets, ouvr. cité, p. 63. 
impulsif) en les plaçant dans un temps du récit distinct : l'ensemble des dialogues et des récits est rapporté par un narrateur homodiégétique («mon père»), qui s'adresse lui-même à un auditoire («mes enfants»). Cette situation met en lumière l'analogie entre la situation du moi-narrant et du père de famille, ce qui souligne la construction en abyme du conte. Ces décalages permettent au lecteur de prendre une distance critique à l'égard des discours qui sont prononcés et de les replacer dans leur contexte.

À la première lecture, le lecteur adhère au propos du philosophe qui reproche à son père de n'avoir pas écouté son cœur et d'avoir respecté le testament du curé de Thivet, favorable aux riches libraires, au détriment de sa misérable famille. Il s'est laissé persuader par le père Bouin : la décision de ce dernier "ne prouve que l'autorité redoutable des opinions religieuses sur les têtes les mieux organisées, et l'influence pernicieuse des lois injustes, des faux principes sur le bon sens et l'équité naturelle ${ }^{57}$ ", selon le philosophe. Ce dernier semble ainsi s'attacher à démonter les croyances religieuses qui aveuglent aussi bien le père Bouin que l'abbé, le frère du philosophe, au point de leur faire oublier leur piété à l'égard de leurs prochains :

Les juges s'en tiennent strictement à la loi, comme mon père et le père Bouin, et font bien. [...] Ils sacrifient quelquefois contre le témoignage de leur conscience, comme mon père et le père Bouin, l'intérêt du malheureux et de l'innocent qu'ils ne pourraient sauver sans lâcher la bride à une infinité de fripons, et font bien ${ }^{58}$.

Les répétitions mécaniques dans le discours de l'abbé expriment l'aveuglement de sa conscience par ses croyances. Le philosophe semble au contraire prôner le recours à une religion naturelle, qui reposerait sur la raison et l'empathie à l'égard d'autrui. Mais la structure enchâssée met en lumière les décalages entre la "sagesse» que prône le Moi-philosophe et ses démonstrations excessives et provocatrices ${ }^{59}$. Alors qu'il reproche à son père de n'avoir pas écouté son cœur, le philosophe se montre prêt à refuser les soins à un criminel malade et va même jusqu'à adopter des positions de plus en plus absurdes : un fossoyeur qui précipite un scélérat pestiféré du troisième étage vaudrait mieux qu'un médecin qui l'aurait guéri. Le médecin Bissei, attaché à la règle d'Hippocrate, se montre plus humain et

57. Entretien d'un père avec ses enfants, ou du danger de se mettre au-dessus des lois, ouvr. cité, p. 484 .

58. Ibid., p. 485 .

59. A. Strugnell, "Les fonctions textuelles du moi dans deux dialogues philosophiques de Diderot", Studies on Voltaire and the Eighteenth Century, Oxford, Voltaire Foundation, vol. 208, I982, p. 176. 
plus tolérant. Les incohérences des personnages participent à l'élaboration d'un «dialogue naturel ", tel que l'appelait de ses vœux Mirzoza au théâtre. Elles sont aussi l'expression de l'anthropologie philosophique de Diderot et notamment de sa conception du moi-multiple ${ }^{60}$ : pour Diderot, l'unité de l'âme est une illusion, et une illusion dangereuse, responsable des persécutions des dévots intolérants. Le conte se présente là encore comme un laboratoire où se construisent, s'énoncent et s'analysent tous les discours, en particulier ceux qui cherchent à asséner des vérités, alors qu'ils ne sont que l'expression de croyances. Si, dans l'Entretien, le dispositif narratif place le lecteur en situation de spectateur distancié de récits et d'opinions diverses, dans Les Deux Amis de Bourbonne, la structure charge le lecteur d'une fonction de juge, devant lequel les personnages-conteurs viennent déposer plusieurs versions d'une même histoire.

$\grave{A}$ un premier niveau narratif, une narratrice $\left(\mathrm{Mme} \mathrm{de}^{* * *}\right)$ s'adresse à "petit frère", apostrophe qui rappelle la situation épistolaire de la première version (il s'agissait d'une lettre censée être de $\mathrm{M}^{\mathrm{me}}$ de Pruneveaux à Naigeon). Cette voix narrative rapporte l'histoire de Félix et d'Olivier, deux amis inséparables qui ont grandi ensemble, et qui sont prêts à tout l'un pour l'autre ${ }^{6}$. D'abord, elle rapporte ce que lui a transmis la rumeur («on prétend qu'il était fier de cette blessure : pour moi, je n’en crois rien $\left.^{62} »\right)$, puis le témoignage de la femme d'Olivier, qu'elle a rencontrée, entourée de ses quatre enfants, ensuite la version du subdélégué Aubert ("qui est un bon homme, bien rond, et qui nous a envoyé le récit suivant, sur la vérité duquel vous pouvez compter $\left.{ }^{63} »\right)$. Aubert appuie sa version sur le témoignage de la charbonnière, qui a accueilli Félix. Mme de*** annexe à sa présentation la lettre de M. Papin, docteur en théologie, qui donne son point de vue et qui cherche à la convaincre de l'impiété de ces deux familles. Un autre niveau narratif apparaît à la fin, qui englobe la narration de Mme de $e^{* * *}$. Ce narrateur insère un billet que Mme de ${ }^{* * *}$ a envoyé à

\footnotetext{
6o. C. Duflo, ouvr. cité, p. 254-267.

6I. Ils tombent amoureux de la même femme. C'est Olivier qui l'épouse et lui donne des enfants. Félix part sur les routes et se fait contrebandier. Il est arrêté et condamné. Olivier l'apprend, sauve son ami et se fait tuer lorsqu'il organise sa fuite. Félix trouve refuge chez un charbonnier dont la famille l'accueille et le soigne. Le charbonnier meurt sous un coup de feu tiré par un agent de la maréchaussée, qui cherchait à arrêter Félix. Ce dernier parvint à retrouver la maison d'Olivier dont il apprend la mort. Il découvre sa femme dans la misère. Félix organise le mariage entre la fille de la charbonnière et le fils d'Olivier. Il parvient à se faire engager comme garde-chasse par M. de Rançonnières, qui obtient sa grâce, ce qui lui permet d'envoyer de l'argent aux deux veuves.

62. Les Deux Amis de Bourbonne, dans Contes et romans, ouvr. cité, p. 440.

63. Ibid., p. 44I.
} 
M. Papin avec ses aumônes et commente ironiquement le comportement du personnage :

On pense bien que la veuve Olivier et Félix n'eurent aucune part aux aumônes de Mme de***. Félix mourut; et la pauvre femme aurait péri de misère avec ses enfants, si elle ne s'était réfugiée dans la forêt chez son fils aîné où elle travaille, malgré son grand âge, et subsiste comme elle peut, à côté de ses enfants et de ses petits-enfants ${ }^{64}$.

Elle se dit émue par les deux misérables, mais son empathie n'est pas suivie d'effets : elle s'est laissé convaincre par l'abbé, comme le père du philosophe dans l'Entretien. La critique ${ }^{65}$ a souligné une incohérence temporelle : la "grande femme debout avec quatre petits enfants à ses pieds ${ }^{66}$ ", que Mme de ${ }^{* * *}$ a rencontrée au début du texte, semble avoir vieilli bien vite. Mais ces deux parties du texte appartiennent-elles au même cadre narratif et temporel? Est-on toujours dans le récit de Mme de***? Le pronom indéfini fait entendre une autre instance narrative, qui dénonce l'attitude de Mme de***. Il s'agit donc d'un cadre narratif différent de celui dans lequel s'insèrent tous les témoignages, dont celui de $\mathrm{Mme} \mathrm{de}^{* * *}$. Cette idée est corroborée par les temps verbaux : on passe progressivement du système du récit (eurent, mourut, seétait réfugiée), au système du discours (travaille, subsiste). Tout se passe comme si on quittait progressivement le temps du récit, pour rejoindre le temps de l'écriture, comme si on s'éloignait petit à petit du tableau pour en analyser les procédés, ce que confirme la réflexion métapoétique qui clôt le texte. Par conséquent, comme le conte oriental, le conte moral présente quatre niveaux narratifs.

Une telle construction rapproche le texte d'un dossier d'enquête. Comme Mme de***, le lecteur se trouve face aux différentes versions de l'histoire de Félix et d'Olivier, qui s'apparentent à des dépositions : les récits de chaque personnage sont autant de mises en perspective, la vérité de chacun dépend de son point de vue, au sens concret et abstrait du terme. Dès lors, qui croire? Pourquoi accorder davantage de créance au docteur en théologie qu'à la femme d'Olivier? En outre, la structure emboîtée donne au lecteur un recul supplémentaire par rapport à la narratrice. Comme elle, il est placé en situation de juge, mais il est conscient des effets de la rhétorique et du pathos : la réaction de Mme de*** lui en donne un exemple. Au lecteur de détecter les modalisations du discours, signes du jugement et des croyances

64. Ibid., p. 448.

65. J. Ehrard, L'Invention littéraire au XVIII siècle : fictions, idées, société, ouvr. cité, p. I60. Voir également J.-C. Rebejkow, "Quelques réflexions sur la révision des Deux Amis de Bourbonne, par Diderot", Les Lettres romanes, t. L, n 3-4, août-novembre 1996, p. 208.

66. Les Deux Amis de Bourbonne, ouvr. cité, p. 44I. 
des personnages. L'évidence devient dès lors à chaque instant problématique. Comme l'affirme Jean Ehrard, «le récit devient instrument de recherche : une vraie recherche qui ne sait pas d'avance ce qu'elle va trouver, progresse par remises en cause de ce qui semblait acquis, renouvelle de moment en moment non seulement ses hypothèses, mais ses interrogations, au point de faire bien vite oublier son point de départ ${ }^{67}$ ». La fiction devient en ce sens une arme particulièrement efficace pour débusquer toutes les affirmations dogmatiques, et donc toutes les croyances, y compris la fiction elle-même.

En effet, Les Deux Amis de Bourbonne et Les Bijoux indiscrets insèrent au cœur du récit une réflexion métapoétique sur l'illusion romanesque pour le premier, théâtrale, pour le second. À la fin du conte moral, la voix narrative, qui ironise sur l'attitude de $\mathrm{M}^{\mathrm{me}} \mathrm{de}^{* * *}$, compare les différents types de contes. Elle imagine même les réactions du lecteur fictif, qui coopère à son tour à la construction du texte. Cette voix narrative s'apparente ainsi à l'instance qui souligne la structure (titres, parties) dans le conte oriental. Dans Les Bijoux indiscrets, lors de la conversation avec Sélim sur les tragédies contemporaines, c'est Mirzoza qui développe une théorie sur l'illusion théâtrale. Elle reconnaît que «la perfection d'un spectacle consiste dans l'imitation si exacte d'une action, que le spectateur trompé sans interruption s'imagine assister à l'action même ${ }^{68}$ ». Quels sont les procédés capables de créer l'illusion? L'effacement de l'auteur, un dialogue naturel et l'importance accordée aux circonstances. Mirzoza reproche en effet aux pièces classiques de mettre en scène des personnages qui ne sont que des porteparole de l'auteur, des êtres de papier et non de chair : «Messieurs, au lieu de donner à tout propos de l'esprit à vos personnages, placez-les dans les conjonctures qui leur en donnent ${ }^{69}{ }^{\prime}$, dirait-elle aux Modernes. Se trouvent ainsi esquissés les principes de l'esthétique diderotienne qui seront développés aussi bien dans Dorval et moi que dans De la poésie dramatique ${ }^{70}$ et que Diderot applique dans les deux contes moraux étudiés. Tel est le tour de force des contes de Diderot : au moment même où ils créent les conditions nécessaires à l'adhésion du lecteur, ils rompent sans cesse l'illusion et développent une réflexion sur ses mécanismes.

En conclusion, les contes orientaux et moraux de Diderot font entendre différentes opinions, montrent les circonstances dans lesquelles elles sont

67. J. Ehrard, ouvr. cité, p. 169.

68. Les Bijoux indiscrets, ouvr. cité, p. 135 .

69. Ibid., p. 136.

70. «Lillusion est leur but commun [au drame et au roman] : mais d'où dépend l'illusion? Des circonstances. Ce sont les circonstances qui la rendent plus ou moins difficile à produire", De la poésie dramatique, J. Goldzink (éd.), Paris, GF Flammarion, 2005, p. 192. 
proférées et mettent en évidence leurs contradictions avec l'attitude des énonciateurs. Ils relèvent d'une écriture poétique et hiéroglyphique, qui parle à la raison et aux sens, et que Diderot décrit dans la Lettre sur les sourds et muets à l'usage de ceux qui entendent (I75I). L'écriture emblématique se définit comme celle "qui fait que les choses sont dites et représentées tout à la fois; que dans le même temps que l'entendement les saisit, l'âme en est émue, l'imagination les voit, l'oreille les entend ${ }^{71} »$. Diderot affirme par là la capacité du langage poétique à recomposer la densité du réel et à recréer la simultanéité des sensations. L'écriture symbolique du conte devient dès lors un moyen d'éveiller les esprits, plus efficace qu'un traité ou que des arguments, car elle s'adresse à l'homme dans toute sa complexité, à la fois à sa raison et à ses sens, comme Diderot l'affirme dans sa définition de la «leçon», dans l'Encyclopédie : "On formeroit mieux la raison en faisant observer la liaison naturelle des choses $\&$ des idées, qu'en donnant l'habitude de faire des argumens ${ }^{72}$.» Telle est bien l'expérience d' " analogie généralisée ${ }^{73}$ ", que nous font vivre les contes diderotiens, grâce à leur structure enchâssée.

Dès lors, les contes de Diderot offrent au lecteur une expérience de déstabilisation de l'ensemble des discours et lui permettent de saisir une vérité humaine complexe. Ils peuvent donc être considérés comme des textes philosophiques : ils remettent en cause les préjugés, placent le lecteur en situation de doute permanent, tout en montrant les mécanismes de la croyance. Ils ont ainsi la même fonction que les traités philosophiques. Comme l'Essai sur l'entendement humain de Locke, ils nous font expérimenter différents "degrés d'assentiment ${ }^{74}$ », selon l'expression du philosophe anglais, depuis la pleine assurance jusqu'à la conjoncture, au doute et à la défiance. Ils deviennent en ce sens un objet utile au philosophe, un outil de connaissance, compris comment moyen de connaître et d'expérimenter les moyens de la connaissance elle-même. En effet, comme l'affirme Diderot lui-même dans ses Réflexions sur le livre De l'Esprit, par M. Helvétius (1758), qui précède

7I. Diderot, Lettre sur les aveugles : à l'usage de ceux qui voient; Lettre sur les sourds et muets : à l'usage de ceux qui entendent et qui parlent, M. Hobson et S. Harvey (éd.), Paris, Flammarion, 2000, p. II6.

72. Diderot, article "Leçon", dans Encyclopédie, ouvr. cité, vol. I, t. IX, p. 332.

73. A. Gaillard, «Contage et sexualité, le récit à double entente de Diderot, L'Oiseau blanc, conte bleu», dans A. Defrance et J.-F. Perrin (éd.), Le Conte en ses paroles : la figuration de l'oralité dans le conte merveilleux du Classicisme aux Lumières, Paris, Desjonquères, 2007, p. 200.

74. J. Locke, Essai sur l'entendement humain, traduction par P. Coste, Paris, Libraire générale française, 2009, p. 949. 
sa Réfutation ${ }^{75}$, il n'y a rien qui veuille moins être annoncé directement, de manière objective et méthodique, qu'un paradoxe. Il s'agit au contraire de «balloter» le lecteur, du blanc au noir, du noir au blanc, pour qu’il expérimente la complexité du sujet traité. "L'esprit d'invention» apparaît plus à même de penser certaines questions d'ordre philosophique ou moral car il «s'agite, se meut, se remue d'une manière déréglée; il cherche. L'esprit de méthode arrange, ordonne, et suppose que tout est trouvé ${ }^{76} »$.

75. Diderot, Réflexions sur le livre De l'Esprit par M. Helvétius, dans Euvres complètes de Diderot: revues sur les éditions originales... Étude sur Diderot et le mouvement philosophique au XVIII ${ }^{e}$ siècle. T. 2, J. Assézat et M. Tourneux (éd.), Paris, Garnier frères, I875, p. 267-274.

76. Ibid., p. 273. 